\title{
The accumulation of exosome-associated microRNA-1246 and microRNA-150-3p in human red blood cell suspensions
}

Yujie Kong ${ }^{1,2}$, Xue Tian ${ }^{1,2}$, Rui He ${ }^{1,2}$, Chenyue Li ${ }^{1,2}$, Haixia Xu ${ }^{1,2}$, Li Tian $^{1,2^{*}}$ and Zhong Liu ${ }^{1,2^{*}}$

\begin{abstract}
Background: Transfusion-related immunomodulation (TRIM) can be caused by exosomes, in which case, microRNAs (miRNAs) are one critical factor impacting exosome behavior. This study aims to investigate and analyze the expression profiles of exosomal miRNA in red blood cell (RBC) suspensions during storage and to identify potential TRIMrelated miRNAs as well as their potential functions.
\end{abstract}

Methods: A total of 25 packs of RBC suspensions were randomly collected. Exosome were extracted by ultracentrifugation and then identified and characterized by nanoparticle tracking analysis (NTA), transmission electron microscopy (TEM) and western blot (WB). Exosomal miRNA profiles were acquired using gene chips in five packs on week 1 and week 5 . The expression data were compared from the two time points identifying accumulated miRNAs with statistical significance and their predicted targeting genes were analyzed. Based on the gene chip results, quantitative reverse transcription-polymerase chain reactions (qRT-PCR) were performed to verify miRNA accumulation in the rest 20 packs sampling on week 1,3 and 5.

Results: Gene chip analysis revealed that most exosomal miRNAs were enriched as the storage period progressed. Compared to samples from week 1, week 5 samples exhibited a total of 539 differential miRNA expressions, among which, 159 were statistically significant $(P<0.05)$ and $148(93.08 \%)$ were accumulated. In the bioinformatics functional analysis, significant immunoregulatory annotations related to the thyroid hormone, mitogen-activated protein kinase (MAPK), focal adhesion and RAS signaling pathways were identified. The top 17 differential expression miRNAs were validated by qRT-PCR. The results confirmed that all the 17 miRNAs were accumulated with increasing storage time. In particular, miRNA-1246 and miRNA-150-3p were the most enriched strands by more than 150-folds in the 5-week storage period.

Conclusions: As storage progressed, numerous exosomal miRNAs accumulated in the RBC suspensions, which are informatically connected to multiple immuno-signaling pathways. MiRNA-1246 and miRNA-150-3p may be essential mediators impacting the immunoregulation functions of exosomes in RBC suspensions, considering their significant accumulating scales. Further research should therefore focus on the relationship between these miRNAs and TRIM.

Keywords: Exosome, MicroRNA, Red blood cell (RBC) suspensions, Transfusion-related immunomodulation (TRIM)

\footnotetext{
*Correspondence: 241775291@qq.com; liuz@ibt.pumc.edu.cn ${ }^{1}$ Clinical Transfusion Research Center, Institute of Blood Transfusion, Chinese Academy of Medical Sciences and Peking Union Medical College, 26 Huacai Rd, Longtan Industry Zone, Chenghua District, Chengdu 610052, Sichuan Province, People's Republic of China Full list of author information is available at the end of the article
}

\section{Background}

Red blood cell (RBC) suspension is commonly transfused in clinic for patients suffering from anemia. It can be stored for 35 days at $4{ }^{\circ} \mathrm{C}$ in an additive solution with A-form of acid-citrate-dextrose (ACD-A). The storage time can be further extended to 42 days with saline 
adenine glucose mannitol (SAG-M) [1]. During storage, RBCs undergo a series of morphological and biochemical changes known as RBC storage lesions, which affect the quality and functions of RBCs [2]. The impact of the transfusion of RBCs with different storage period on individuals' clinical outcomes has always been an active and controversial topic in the clinical community [3]. Studies have reported that prolonged RBC storage may increase the occurrence of complications and adverse clinical outcomes. Czubak-Prowizor et al. [4] reported that patients with cardiovascular disorders who received "older" RBC transfusions may suffer thrombotic complications. For trauma patients, prolonged RBC storage may increase the risk of deep vein thrombosis and bacterial infections $[5,6]$. These complications are considered to be related to the changes in both RBCs and bioactive substances accumulated during storage [7].

Transfusion-related immunomodulation (TRIM) emerged as a potential explanation for these clinical complications. TRIM refers to certain immune-related responses caused by blood transfusion, some of which can affect the progression and prognosis of an existing disease. TRIM was first described as an immunosuppressive function in renal transplantation patients receiving perioperative blood transfusions [8]. TRIM was subsequently reported to be associated with increased tumor incidence, pathogen infection and mortality [9-11]. Adverse clinical outcomes caused by transfusing longtime stored RBCs have also been reported to be related to TRIM. The mechanisms of TRIM, however, remain largely unknown, and this is especially true of how stored RBC transfusions interact with the recipients' immune system and what are the regulating cascades post transfusion [12].

Exosomes, a type of bioactivity mediator, are consistently produced throughout RBC suspension storage, which function as a tool of intercellular communication and molecule transport [13]. The generation of exosomes is thought to be related to TRIM in blood recipients [14]. Studies have reported that exosomes from RBC suspensions are capable of promoting inflammatory cytokine secretion and mediating $\mathrm{T}$-cell proliferation responses [15]. Understanding the mechanism of RBC suspensionderived exosomes involved in TRIM, however, remains a challenge that requires further investigation. Previous research on exosomes mainly focused on their protein content. Recently, exosomal miRNAs have caught wide attention for their regulatory functions in gene expression, such as their ability to inhibit the translation of target genes [16]. MiRNA can be encapsulated in exosomes and stably exists in the recipient's circulatory system. These exosomes can also fuse to recipient cells delivering exogenous contents, such as miRNAs [17]. MiRNAs act as crucial regulators in many biological processes, such as cell differentiation, signal transduction and immunomodulation [18].

This study focuses on the miRNA contained in exosomes and intends to analyze the dynamic alterations in the expression of exosomal miRNA in RBC suspensions during the storage period. A following bioinformatics analysis was conducted to predict the potential functions associated with immunoregulation in predominantly accumulated miRNAs. Further in vitro and in vivo studies will be performed to investigate the impact of the most accumulated miRNA on TRIM.

\section{Materials and methods Study samples}

Twenty-five packs of randomly selected non-leukoreduced RBC suspensions with anticoagulants (ACD-A) were obtained at the Central Blood Bank of Deyang City, Sichuan province, China. Exosomes extracted from five packs underwent gene chip analysis while the rest exosome samples were collected for quantitative reverse transcription-polymerase chain reaction (qRT-PCR) validation based on gene chip analysis results. This research protocol was approved by the ethics committee of the Institute of Blood Transfusion of the Chinese Academy of Medical Sciences.

\section{RBC suspension storage, exosome isolation and purification}

$\mathrm{RBC}$ suspensions were split into $100 \mathrm{~mL}$ aliquots in 100$\mathrm{mL}$ transfer bags and stored at $4{ }^{\circ} \mathrm{C}$. The aliquots were then subjected to exosome extraction at week 1,3 , and 5 during storage. The aliquots were first centrifuged at $3000 \mathrm{~g}$ for $20 \mathrm{~min}$ to obtain supernatants. Exosomes were isolated from the supernatants by ultracentrifugation. The protocol includes a $10-\mathrm{min}$ initial stage at $300 \mathrm{~g}$, a second 10 -min stage at $2000 \mathrm{~g}$, a 30 -min supernatant separation stage at $10,000 \mathrm{~g}$ and a final 70 -min exosome separation stage at $1,00,000 g$ for twice. The exosome precipitate was resuspended in $200 \mu \mathrm{L}$ of phosphatebuffered saline (PBS). Then, 0.22- $\mu \mathrm{m}$ centrifugal filters (Millipore, USA) were applied to remove large extracellular vesicles (EVs).

\section{Exosome identification}

The extracted exosomes from RBC supernatants were verified by nanoparticle tracking analysis (NTA), transmission electron microscopy (TEM) and western blot analysis (WB).

The particle size distribution was measured by NTA using the NanoSight NS300 instrument (Malvern Instruments, UK). The sizes of the particles were analyzed using DTS v5.10 software (Malvern Panalytical, 
$\mathrm{UK})$, and the results are presented in a particle size distribution graph.

For TEM analysis, copper grids were placed in exosome suspensions and fixed by $2 \%$ paraformaldehyde overnight. The morphology of the isolated exosomes was captured by TEM (FEI Tecnai ${ }^{\mathrm{TM}}$ G2 Spirit, Czech Republic) at $80 \mathrm{kV}$.

In WB analysis, exosomes were first treated with a RIPA lysis buffer, and the protein concentration was calculated using the bicinchoninic acid (BCA) method. Absorbance values were detected by Varioskan LUX (Thermo Fischer Scientific, USA). Exosome protein markers against TSG101 (Abcam, UK), CD9 (Abcam, UK) and CD63 (Santa Cruz, USA) were validated via WB analysis. Protein markers against Calnexin were also detected as a negative control. Lysates of Hela cells were tested as control samples. Signals of the membranes were captured and imaged by ChemiScope Mini 3000 (CLINX, Shanghai).

\section{RNA isolation and CDNA preparation}

The Total RNA was extracted from exosomes using the QRIzol reagent and the miRNeasy Mini Kit (Qiagen, Germany). The quantity and quality of the total extracted RNA were measured by using the Agilent 4200 platform and the Qubit 2.0 Fluorometer (Thermo Fisher Scientific, USA). Only high-quality RNA samples were used for subsequent experiments (total RNA $\geq 0.5 \mu \mathrm{g}, \mathrm{OD}_{260 / 280}=1.9-2.1$ and RNA concentration $\geq 20 \mathrm{ng} / \mu \mathrm{L}$ ). The miScript II RT Kit with a miScripthispec buffer (Qiagen, Germany) was used to prepare cDNA for subsequent gene chip and qRT-PCR validation.

\section{MiRNA profiling}

Microarray analysis was performed by Gminix Biotechnology Company (Shanghai, China) using the Affymetrix Gene Chip miRNA 4.0 array (Affymetrix, USA). Gene chips were then scanned using the Gene Chip ${ }^{\circledR}$ Scanner 3000 7G (Affymetrix). CEL-files of the raw data were exported and then uploaded to the Gminix-Cloud Biotechnology Information (GCBI) website of Genminix Informatics Co., Ltd. (Shanghai, China; http://www. gcbi.com.cn) for further analysis. The data were analyzed applying the robust multichip analysis algorithm (RMA) with Affymetrix default analysis settings and global scaling normalization. The successive approximation method (SAM) was then used for differentially expressed miRNA analysis. According to the filter condition |fold change| $>1.2$ and $P<0.05$, the final differential results were generated.

\section{Bioinformatics analysis of differentially accumulated exosomal miRNA}

The target genes of various differentially increased exosomal miRNA were predicted by miRBase and TargetScan. A gene ontology (GO) analysis of target genes was performed using $P<0.05$ to define statistically enriched GO categories. Pathway analysis was used to determine the significant pathways of the differential genes according to the Kyoto encyclopedia of genes and genomes database (http://www.genome.jp/kegg/). A miRNA-genes network was built to show the interactions of various strands of miRNA with their target genes.

\section{Identification of miRNA expression by qRT-PCR}

The gene chip results suggested 17 top miRNAs that were accumulated more than ten-fold at week 5 compared to week 1 of storage time. Their expression changes in the rest 20 packs were validated by qRT-PCR. qRT-PCR was performed following the manufacturer's miScript SYBR PCR kit (Qiagen, Germany) protocol with different miRNA primers for each cDNA sample of reverse transcription. The miRNA qPCR primers were synthesized by RIBOBIO Biotech (Guangzhou, China). And CelmiRNA-39 (Qiagen, Germany) was used to normalize potential sample-to-sample variations and technical variations. Relative miRNA changes were calculated using the $2^{-\Delta \Delta C T}$ method.

\section{Results}

Analysis and characterization of exosomes derived from $R B C$ suspensions

NTA, TEM and WB were used to identify and analyze the characterization of exosomes isolated from RBC suspensions. NTA particle size distribution analysis demonstrates that the isolated vesicles at week 5 presented the typical exosome size with particle diameters ranging from 30 to $150 \mathrm{~nm}$ (Fig. 1a). The average diameter of the articles was $76.07 \pm 13.65 \mathrm{~nm}$. More than $95 \%$ of the particles falls below the $120 \mathrm{~nm}$ diameter range, and $99.98 \%$ were below $150 \mathrm{~nm}$. TEM confirmed the exosome morphology to be cup-shaped particles (Fig. 1b). Exosomal surface markers CD9, CD63 and TSG101 were tested positive in WB with negative Calnexin marker readings (Fig. 1c).

\section{Comparison of differentially accumulated exosome-associated microRNA during RBC suspension storage}

The gene chip identified a total of 539 miRNAs during the 5 -week storage. Figure $2 \mathrm{a}$ and $\mathrm{b}$ demonstrate information regarding group-specific signal intensities of the exosomal miRNA profile and the volcano plot between week 1 and 


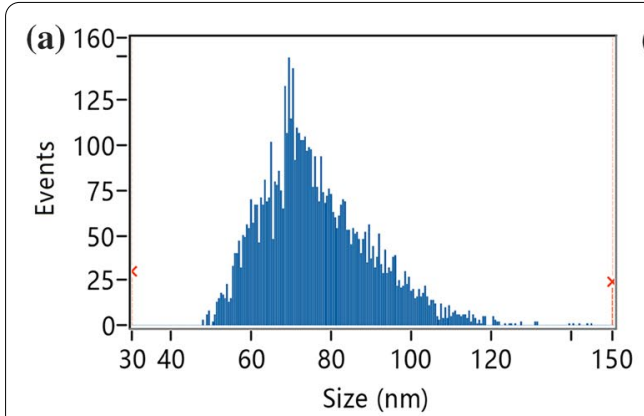

(b)

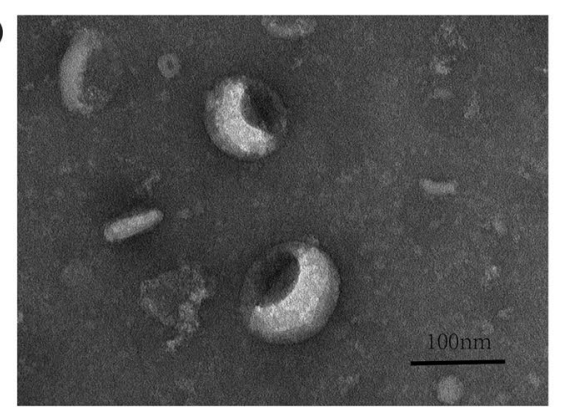

(c) Exosomes Cell lysate

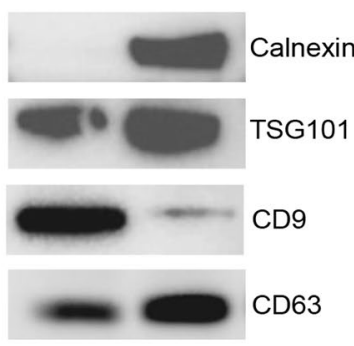

Fig. 1 Characterization of exosomes isolated from RBC suspensions at week 5. a NTA for the particle size distribution of exosomes. b TEM for the morphology of exosomes. Scale bar, $100 \mathrm{~nm}$. cWB identified the specific immunological markers TSG101, CD9 and CD63 of the exosomes

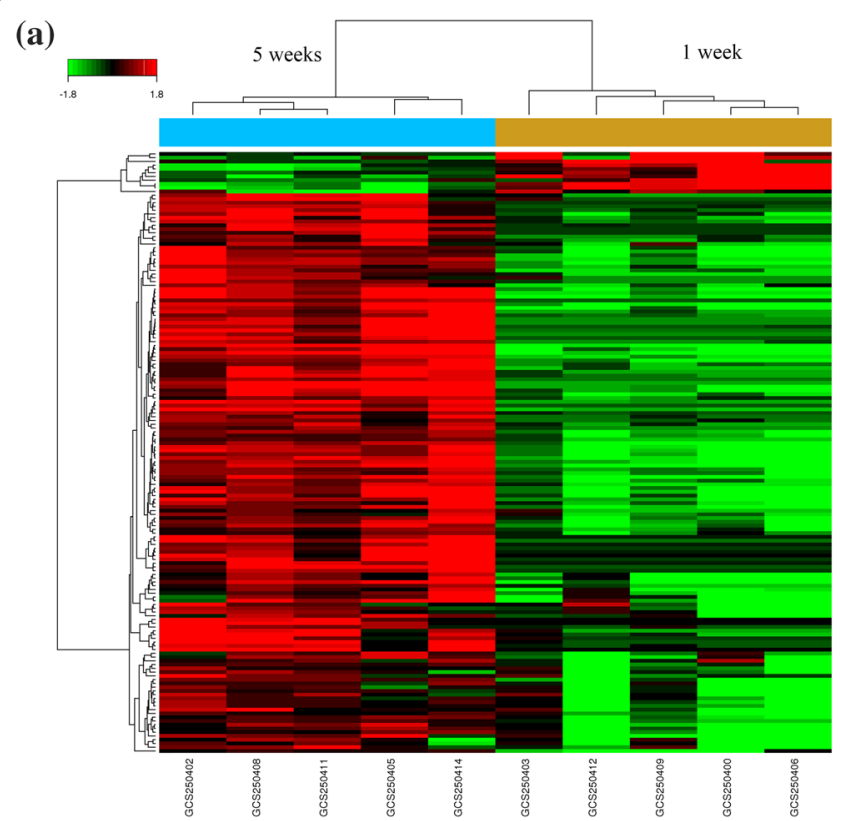

(b)

Fig. 2 Microarray assay of differential miRNA in exosomes derived from RBC suspensions. a Heatmap of differential miRNA expressed in exosomes stored for week 5 vs. week 1. Each column represents an RBC suspension sample, and each row represents a kind of miRNA. The color describes the expression level of each miRNA in each sample, where red indicates a high level of expression and where green indicates a low level of expression. b Volcano plot shows the distribute expression of differential miRNAs. Different colors correspond to the levels of expression, where red represents significantly differential miRNA $(P<0.05)$

week 5. Statistical analysis revealed 159 miRNAs changed differentially (148 increased and 11 decreased, |fold change $>1.2$ and $P<0.05)$, 59 of which were accumulated more than fivefold at week 5 compared to week 1 . Table 1 and Additional file 1: Table S1 list the mean signal, fold changes and $P$ values of the 59 miRNAs at week 1 and week 5.
Bioinformatic analysis: GO function enrichment and KEGG pathway of predicted target genes of significantly differentially accumulated miRNAs

To further determine the functions of the significantly accumulated miRNAs, the miRanda and Targetscan databases were used to predict targeting genes of these miRNAs, suggesting 5538 non-repeated genes. 
Table1 List of differential exosomal miRNAs accumulated more than tenfold at week 5 vs. week 1 storage time of RBC suspensions

\begin{tabular}{|c|c|c|c|c|}
\hline miRNA & $\begin{array}{l}\text { week } 5 \log _{2} \\
\text { mean signal }\end{array}$ & $\begin{array}{l}\text { week } 1 \log _{2} \\
\text { mean signal }\end{array}$ & $\begin{array}{l}\text { week } 5 \text { vs. } \\
\text { week } 1 \text { fold } \\
\text { change }\end{array}$ & $P$ value \\
\hline $\begin{array}{l}\text { hsa-miR- } \\
\text { 6824-5p }\end{array}$ & 6.3266 & 1.7901 & 23.2080 & 0.0019 \\
\hline $\begin{array}{l}\text { hsa-miR- } \\
6716-5 p\end{array}$ & 7.7924 & 3.3319 & 22.0159 & 0.0022 \\
\hline hsa-miR-1246 & 8.4004 & 3.9677 & 21.5962 & 0.0031 \\
\hline $\begin{array}{l}\text { hsa-miR- } \\
\text { 939-5p }\end{array}$ & 6.9238 & 2.6953 & 18.7462 & 0.0020 \\
\hline $\begin{array}{l}\text { hsa-miR- } \\
4433-3 p\end{array}$ & 7.4835 & 3.2970 & 18.2076 & 0.0021 \\
\hline $\begin{array}{l}\text { hsa-miR- } \\
\text { 4701-3p }\end{array}$ & 6.6046 & 2.5412 & 16.7191 & 0.0026 \\
\hline $\begin{array}{l}\text { hsa-miR- } \\
\text { 6849-5p }\end{array}$ & 5.9334 & 1.9205 & 16.1438 & 0.0021 \\
\hline hsa-miR-595 & 5.7246 & 1.9235 & 13.9395 & 0.0024 \\
\hline $\begin{array}{l}\text { hsa-miR- } \\
150-3 p\end{array}$ & 6.0808 & 2.2974 & 13.7697 & 0.0023 \\
\hline $\begin{array}{l}\text { hsa-miR- } \\
\text { 7844-5p }\end{array}$ & 5.6850 & 1.9356 & 13.4488 & 0.0023 \\
\hline $\begin{array}{l}\text { hsa-miR- } \\
\text { 6798-5p }\end{array}$ & 8.3634 & 4.6340 & 13.2639 & 0.0025 \\
\hline hsa-miR-4689 & 7.9570 & 4.2640 & 12.9339 & 0.0031 \\
\hline $\begin{array}{l}\text { hsa-miR- } \\
\quad 1180-3 p\end{array}$ & 6.1168 & 2.4509 & 12.6924 & 0.0028 \\
\hline $\begin{array}{l}\text { hsa-miR- } \\
\text { 3064-5p }\end{array}$ & 5.4298 & 1.8060 & 12.3269 & 0.0024 \\
\hline $\begin{array}{l}\text { hsa-miR- } \\
1225-5 p\end{array}$ & 6.5773 & 3.0099 & 11.8550 & 0.0029 \\
\hline $\begin{array}{l}\text { hsa-miR- } \\
\quad 6819-5 p\end{array}$ & 5.6768 & 2.2801 & 10.5317 & 0.0027 \\
\hline hsa-miR-1268b & 7.3709 & 4.0387 & 10.0714 & 0.0037 \\
\hline
\end{tabular}

$\mathrm{GO}$ analysis described the function of the predicted targeting genes, as the GO database mainly includes biological pathways, cellular components, and molecular functions. The results of GO overexpression analysis indicate that the genes were primarily involved in RNA polymerase II transcription cofactor activity, chromatin DNA binding, transcription factor activity and RNA polymerase II transcription factor binding (biological pathways). They were also primarily involved in the presynaptic active zone, the presynaptic membrane and the phosphatase complex (cellular components) as well as in the negative regulation of neurogenesis, the negative regulation of nervous system development and the negative regulation of cell development (molecular functions) (Fig. 3a). KEGG pathway analysis shows that the most significant pathway was the thyroid hormone signaling pathway (Channel ID: 04919), which was correlated with growth, development and metabolism. The target genes of the differential miRNA, however, were mainly enriched in the MAPK signaling pathway (Path ID: 04010), which played important roles in various cellular functions (Fig. 3b). Other pathways were significantly enriched, including focal adhesion (Path ID: 04510) and the Ras signaling pathway (Path ID: 04014). Figure 4 describes the network of the top 17 accumulated miRNAs, together with their targeting genes.

\section{Verification of miRNAs expression by qRT-PCR}

The expression trend of the top 17 miRNAs were validated by qRT-PCR at week 1,3 and 5 , in 20 of the 25 packs of RBCs. The results are presented in relative expression changes, confirming all 17 miRNAs were increased across storage period. From week 1 to week 3,10 miRNAs accumulated statistically significant, among which miRNA-1246, miRNA-150-3p and miRNA-3064-5p increased more than tenfold. The most abundant miRNA at week 3 was miRNA-150-3p with an average change of 73.67 -fold against week 1 background.

A total of 11 miRNAs accumulated significantly from week 3 to week 5, among which miRNA-1246 exhibited the most change.

Comparing data at week 5 and week 1 , all candidates experienced statistically significant changes $(P<0.01)$. The average changes in six miRNAs were more than tenfold: miRNA-1246, miRNA-150-3p, miRNA-3064-5p, miRNA-1225-5p, miRNA-6849-5p and miRNA-4701-3p (high to low in relative expression changes). MiRNA1246, miRNA-1225-5p and miRNA-6849-5p were predominantly accumulated at week 5 . Among these miRNAs, there were two that accumulated by more than 150-folds at week 5 compared to week 1 . On average, miRNA-1246 increased 275.94-folds, and miRNA150-3p increased 150.93-folds (Fig. 5).

\section{Discussion}

Despite RBCs' rapid application in clinical transfusion, they may raise adverse reactions in recipients, such as acute hemolytic transfusion reactions and delayed hemolytic (or serologic) transfusion reactions [19]. Sufficient evidence now supports the connection between RBC transfusion and immunomodulation via various mechanisms. The recent interest in the relationship between TRIM and extracellular vehicles from blood components has raised heated discussion in the academic community $[20,21]$. Some studies suggest that exosomes in the RBC units may promote TRIM by pro-inflammatory cytokines and immune activation [12, 15]. However, such notion requires further illumination. Recent publications suggest a range of miRNAs in exosomes are involved in 
(See figure on next page.)

Fig. 3 Prediction of the functions and signal pathways of target genes for significant accumulated exosomal miRNA via GO and KEGG pathway analysis. a The GO function enrichment terms of target genes for the significantly accumulated miRNA sat week 5 vs. week 1 of storage time; the function terms were ranked from high to low by enrichment score. $\mathbf{b}$ The KEGG pathway analysis of the target genes for the significantly accumulated miRNAs at week 5 vs. week 1 of storage time. The dot color represents $-\log (P$ value), and the dot size represents the number of target genes included in the pathway

the regulation of immunity, including the innate immunity, the development of $\mathrm{T}$ and $\mathrm{B}$ cells, and proliferation of monocytes and neutrophils [22, 23]. In this study we sought to explore the exosomal miRNAs as potential functional molecules impacting TRIM. The miRNA profiles of supernatant exosomes from non-leukoreduced $\mathrm{RBC}$ suspensions were characterized and analyzed across a 5-week storage period and the significantly accumulated miRNAs were identified and annotated according to the function of their predicted targeting genes.

One similar study analyzed the miRNA profiles of exosomes collected from leukoredced RBC units in three separate samples obtained on day 14 of storage [24]. Compared to the in-data-base (miRDeep) 287 miRNAs previously reported in mature erythrocytes [25], the cross results revealed that 78 miRNAs were both expressed in all exosome samples and mature erythrocytes. The top three enriched miRNAs were miRNA-125b-5p, miRNA4454 miRNA-451a, and these miRNAs were validated by qRT-PCR. The results show higher abundance of miRNA-451a than others and the expression increased with extending storage time, on average, 7.58-folds of increase across a 5-week storage period. This result is different from ours, which may be due to its comparison of miRNA expression between single miRNA profiling time point at day 14 and the previous identified miRNA in mature RBC. Our study, on the other hand, set different profiling points across the 5 weeks storage, ensuring the screening of miRNA with constant increase. Notably, we've identified several miRNAs with changing accumulation curves, further addressed the notion that single profiling time point may limit screening results.

In addition, our study identified miRNAs with a larger increase scale at more than 150 -folds. This may be caused by the different selection of sampling source. We adopted non-leukoreduced RBC suspensions as the exosome source, considering its predominant occupation of the clinical transfusion market. In this sense, the exosome-associated miRNAs profiled in this study may not be limited to single RBC source but also other cells in the suspension. We predict the most enriched exosomal miRNA across the storage period might impact the quality of RBC suspensions before transfusion. This notion, however, requires further investigation.

GO and KEGG were used to predict the potential functions of the target genes of the statistically abundant types of miRNA within 5 weeks of storage time. The results indicate that the encoded proteins mainly came from synapse-related proteins and phosphatase complexes (presynaptic active zone, presynaptic membrane, phosphatase complex and protein serine/threonine phosphatase complex) that had transcription factor activities and DNA binding functions involved in the development of different systems, hormone secretion and transportation. The KEGG database was used to further annotate the target genes, revealing significant enrichment in the thyroid hormone signaling pathway. The thyroid hormone is closely correlated with immunoregulation and plays an essential role in several inflammatory-related processes, such as the production of cytokines and chemokines [26]. The MAPK signaling pathway experienced the greatest enrichment of its target genes. The MAPK signaling pathway contains three main kinases, namely the MAPK kinase kinase, the MAPK kinase and MAPK. They activate and phosphorylate downstream proteins to regulate and control various physiological and pathological functions, including innate immunity, inflammation, apoptosis, cell growth, cell differentiation, tumor invasion and metastasis, among others [27, 28]. In addition, focal adhesion and the Ras signaling pathway were also significantly enriched, both of which play important roles in immunomodulation. Focal adhesion participates in multiple biological processes including cell proliferation and the regulation of gene expression, and it serves as a scaffold for many signaling pathways [29]. The Ras protein with GTPase activity acts as a switch protein in the cell signaling pathway, regulating cell differentiation, proliferation, apoptosis and survival, among other activities [30]. Recent research has revealed that RAS plays a vital role in the formation of exosomes, and it may play a signaling role through exosome secretion [31]. The target genes of miRNAs accumulated during storage were significantly enriched in the above signaling pathways, and these signaling pathways played important roles in the immune system. Therefore, by regulating these signaling pathways, the exosomal miRNAs enriched in old RBC suspensions could be involved in immunoregulation.

RBC suspensions stored for 5 weeks had 17 significantly different miRNAs accumulated more than tenfold compared with those of RBC suspensions stored for 1 week. Afterward, qRT-PCR identification results show 
(a)

(b)

molecular function

Diffgene_count

- 10

20

\section{ifgene Sig Go}
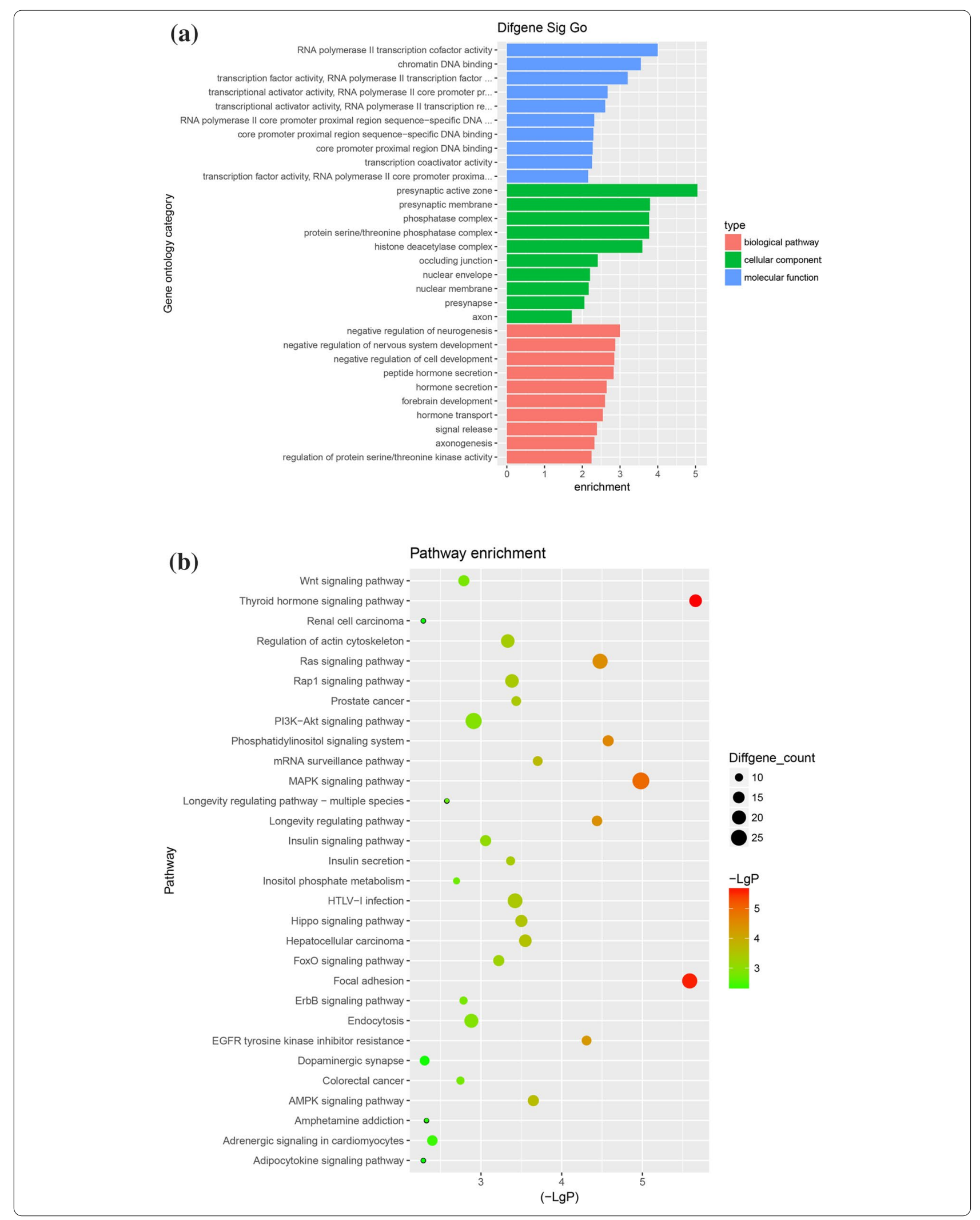


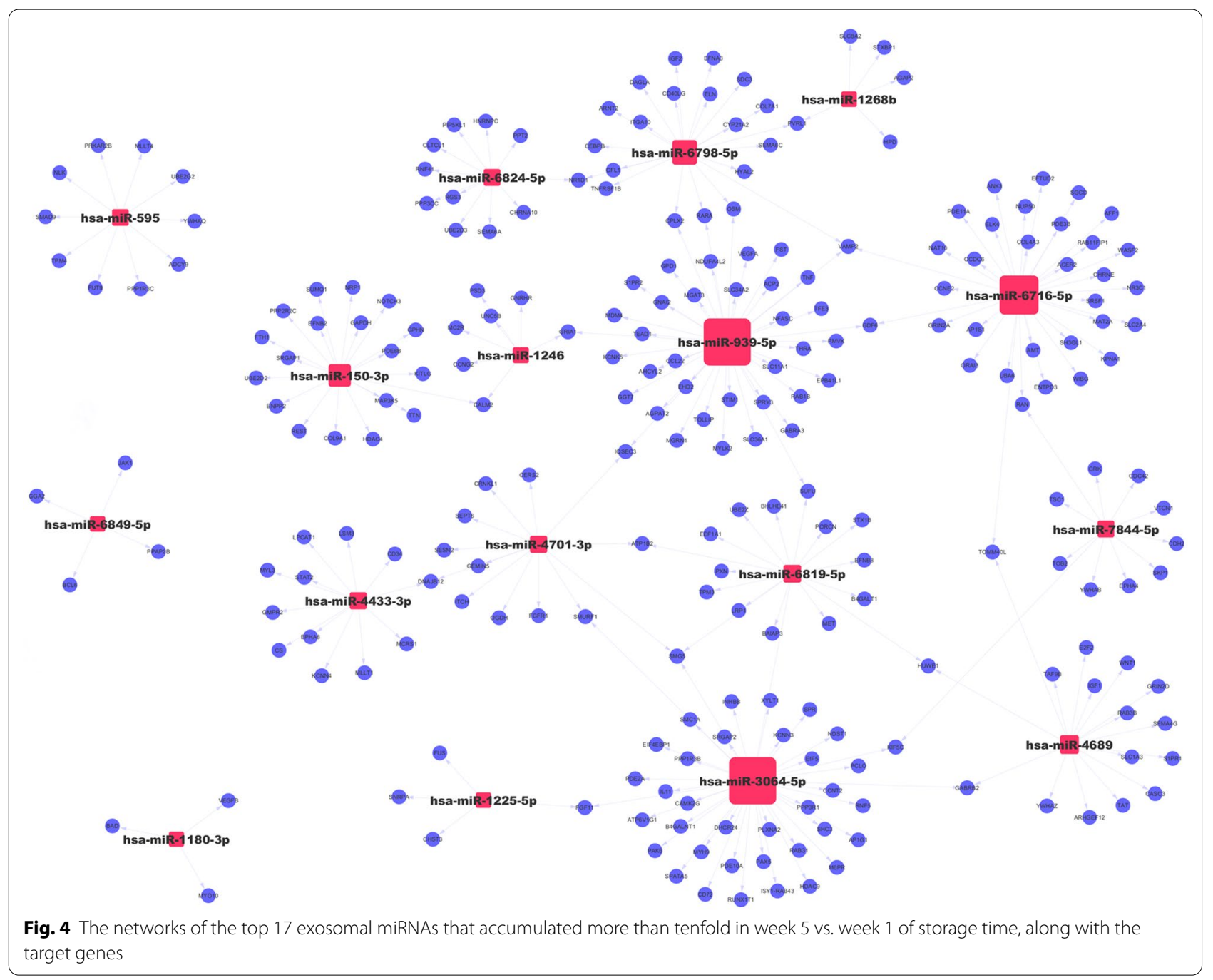

that there were six miRNAs accumulated more than tenfold at week 5 compared to week 1 storage. The six most accumulated miRNAs are partly reported to play potential roles in immuno-regulation. For instance, in dilated cardiomyopathy (DCM), miRNA-3064-5p, miRNA6849-5p and miRNA-4701-3p are upregulated in abnormal activated CD4 $+\mathrm{T}$ cells, which may be associated with the proliferation of CD4 $+\mathrm{T}$ cells [32]. The Infusion of these exosomal miRNAs in long-time stored RBC transfusion may participate in the regulation of $\mathrm{CD} 4+\mathrm{T}$ cell-mediated inflammatory and immune responses. Ranked by relative expression changes compared to week 1, miRNA-1246 and miRNA-150-3p were the two most abundant miRNAs both at week 3 and week 5 . Exosomederived miRNA-1246 in hypoxic glioma has been proven to be related to the induction of M2 macrophage polarization by targeting TERF2IP [33]. In addition, miRNA1246 also mediates lipopolysaccharide (LPS)-induced lung injury and neutrophil activation by targeting angiotensin-converting enzyme 2 (ACE2) [34]. MiRNA$150-3 p$ has been reported to play an important role in the anti-apoptosis process [35] and is also involved in inflammation pathways [34]. By inhibiting miRNA-150-3p expression, sacubitril/valsartan can effectively alleviate cyclophosphamide-induced lung inflammation [36]. Collectively, these two miRNAs (over 150-folds at week 5) are both associated with pro-inflammatory responses and might promote transfusion-related acute lung injury (TRALI). The transfusion of long-time stored RBC suspensions with these enriched exosome-associated miRNAs may have a potential role in individual immunoregulatory responses. However, their associations with TRIM or transfusion-related adverse reactions still needs further investigation. 

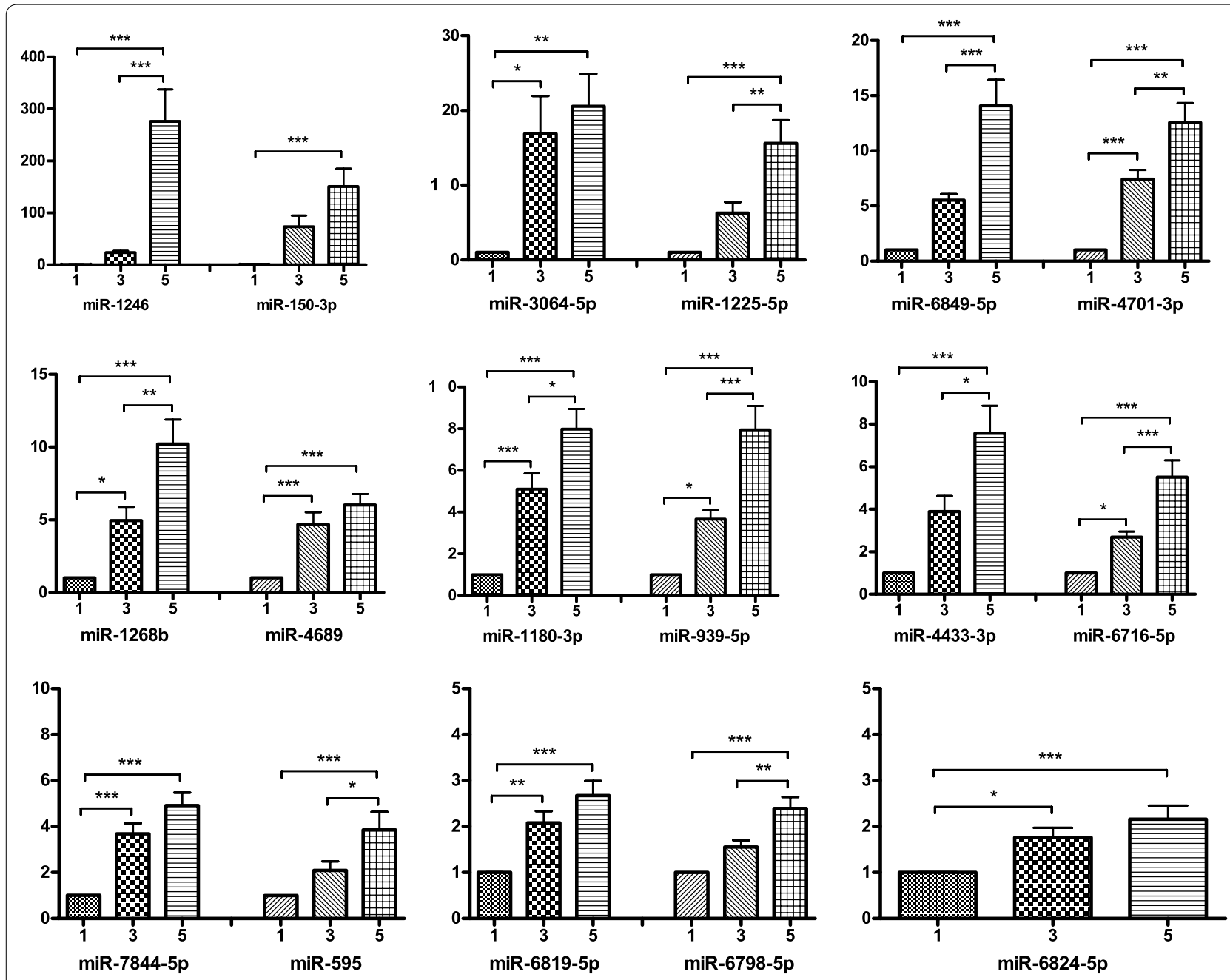

Fig. 5 Validation of exosomal miRNA accumulated more than tenfold at week 5 vs. week 1 storage time $\left({ }^{*} P<0.05,{ }^{* *} P<0.01\right.$ and $\left.{ }^{* * *} P<0.001\right)$. The horizontal axis represents storage time, with week 1 at 1 , week 3 at 3 and week 5 at 5 . The vertical axis represents relative expression changes

\section{Conclusions}

This study revealed that many exosome-associated miRNAs existed in RBC suspensions and can accumulate during storage. Analysis of these exosome-associated miRNAs indicates that they may possess multiple functions during storage or post transfusion. The most enriched miRNA-1246 and miRNA-150-3p, in particular, might be essential mediators of immunoregulation. Following research on the mechanism of how these miRNAs impacting TRIM is to be conducted next.

\section{Abbreviations}

TRIM: Transfusion-related immunomodulation; RBC: Red blood cell; miRNA MicroRNA; NTA: Nanoparticle tracking analysis; TEM: Transmission electron microscopy; WB: Western blot; qRT-PCR: Quantitative reverse transcriptionpolymerase chain reaction; MAPK: Mitogen-activated protein kinase; ACD-A: A-form of acid-citrate-dextrose; SAG-M: Saline adenine glucose mannitol; PBS: Phosphate-buffered saline; EV: Extracellular vesicle; BCA: Bicinchoninic acid; RMA: Robust multichip analysis; SAM: Successive approximation method;
GO: Gene ontology; KEGG: Kyoto encyclopedia of genes and genomes; DCM: Dilated cardiomyopathy; LPS: Lipopolysaccharide; ACE2: Angiotensin-converting enzyme 2 .

\section{Supplementary Information}

The online version contains supplementary material available at https://doi. org/10.1186/s12967-021-02887-2.

Additional file 1: Table S1. List of differential exosomal miRNAs accumulated more than five-fold at week 5 vs. week 1 storage time of RBC suspensions.

\section{Acknowledgements}

None.

\section{Authors' contributions}

YK was responsible for experiment performance, data collection and analysis, and original draft written. $\mathrm{XT}, \mathrm{RH}, \mathrm{CL}$ and $\mathrm{HX}$ were responsible for data curation 
and analysis; LT and ZL contributed to the study design and manuscript revision. All authors read and approved the final manuscript.

\section{Funding}

This work was supported by the CAMS Innovation Fund for Medical Sciences (Grant Nos. 2016-I2M-3-024, 2020-I2M-CoV19-006), Fundamental Research Funds for the Central Universities (Grant No. 3332019170) and Science and Technology Department of Sichuan Province (Grant No. 2020YFH0024).

\section{Availability of data and materials}

The datasets used and/or analyzed during the current study are available from the corresponding author on reasonable request.

\section{Declarations}

\section{Ethics approval and consent to participate}

The research was approved by the ethics committee of the Institute of Blood Transfusion, Chinese Academy of Medical Sciences.

\section{Competing interests}

The authors have disclosed no competing interest.

\section{Author details}

'Clinical Transfusion Research Center, Institute of Blood Transfusion, Chinese Academy of Medical Sciences and Peking Union Medical College, 26 Huacai Rd, Longtan Industry Zone, Chenghua District, Chengdu 610052, Sichuan Province, People's Republic of China. ${ }^{2}$ Key Laboratory of Transfusion Adverse Reactions, CAMS, 26 Huacai Rd, Longtan Industry Zone, Chenghua District, Chengdu 610052, Sichuan Province, People's Republic of China.

Received: 8 January 2021 Accepted: 17 May 2021

Published online: 27 May 2021

\section{References}

1. Hess JR. An update on solutions for red cell storage. Vox Sang. 2006;91(1):13-9.

2. García-Roa M, Del Carmen V-A, Bobes AM, Pedraza AC, González-Fernández A, Martín MP, et al. Red blood cell storage time and transfusion: current practice, concerns and future perspectives. Blood Transfus. 2017;15(3):222-31.

3. Yoshida T, Prudent M, D'alessandro A. Red blood cell storage lesion: causes and potential clinical consequences. Blood Transfus. 2019;17(1):27-52

4. Czubak-Prowizor K, Rywaniak J, Zbikowska HM. Red blood cell supernatant increases activation and agonist-induced reactivity of blood platelets. Thromb Res. 2020;196:543-9.

5. Spinella PC, Carroll CL, Staff I, Gross R, Quay JM, Keibel L, et al. Duration of red blood cell storage is associated with increased incidence of deep vein thrombosis and in hospital mortality in patients with traumatic injuries. Crit Care. 2009;13(5):R151.

6. Juffermans NP, Vlaar AP, Prins DJ, Goslings JC, Binnekade JM. The age of red blood cells is associated with bacterial infections in critically ill trauma patients. Blood Transfus. 2012;10(3):290-5.

7. Sparrow RL. Red blood cell storage and transfusion-related immunomodulation. Blood Transfus. 2010;8(Suppl 3):s26-30.

8. Opelz G, Sengar DP, Mickey MR, Terasaki PI. Effect of blood transfusions on subsequent kidney transplants. Transplant Proc. 1973;5(1):253-9.

9. Blajchman MA. Transfusion immunomodulation or TRIM: what does it mean clinically? Hematology. 2005;10(Suppl 1):208-14.

10. Xu D, Fang X, Li Y, Zhang Z, Li Q. Perioperative blood transfusion is one of the factors that affect the prognosis of gastric cancer. J BUON. 2018;23(3):672-7.

11. Vamvakas EC. Pneumonia as a complication of blood product transfusion in the critically ill: transfusion-related immunomodulation (TRIM). Crit Care Med. 2006;34(5 Suppl):S151-9.

12. Remy KE, Hall MW, Cholette J, Juffermans NP, Nicol K, Doctor A, et al. Mechanisms of red blood cell transfusion-related immunomodulation. Transfusion. 2018;58(3):804-15.

13. Wortzel I, Dror S, Kenific CM, Lyden D. Exosome-mediated metastasis: communication from a distance. Dev Cell. 2019:49(3):347-60.
14. Almizraq RJ, Seghatchian J, Acker JP. Extracellular vesicles in transfusion-related immunomodulation and the role of blood component manufacturing. Transfus Apher Sci. 2016;55(3):281-91.

15. Danesh A, Inglis HC, Jackman RP, Wu S, Deng X, Muench MO, et al. Exosomes from red blood cell units bind to monocytes and induce proinflammatory cytokines, boosting T-cell responses in vitro. Blood. 2014;123(5):687-96.

16. Zhang J, Li S, Li L, Li M, Guo C, Yao J, et al. Exosome and exosomal microRNA: trafficking, sorting, and function. Genom Proteom Bioinform. 2015;13(1):17-24.

17. Yu X, Odenthal M, Fries JW. Exosomes as miRNA carriers: formation-functionfuture. Int J Mol Sci. 2016;17(12):2028.

18. Bartel DP. MicroRNAs: target recognition and regulatory functions. Cell. 2009;136(2):215-33.

19. Zimring JC, Spitalnik SL. Pathobiology of transfusion reactions. Annu Rev Pathol. 2015:10:83-110

20. Belizaire RM, Prakash PS, Richter JR, Robinson BR, Edwards MJ, Caldwell CC, et al Microparticles from stored red blood cells activate neutrophils and cause lung injury after hemorrhage and resuscitation. J Am Coll Surg. 2012;214(4):648-55.

21. BurnoufT, Chou ML, Goubran H, Cognasse F, Garraud O, Seghatchian J. An overview of the role of microparticles/microvesicles in blood components: are they clinically beneficial or harmful? Transfus Apher Sci. 2015:53(2):137-45.

22. Momen-Heravi F, Bala S. Extracellular vesicles in oral squamous carcinoma carry oncogenic miRNA profile and reprogram monocytes via NF-kappaB pathway. Oncotarget. 2018;9(78):34838-54

23. Njock M, Cheng H, Dang L, Nazari-Jahantigh M, Lau A, Boudreau E, et al. Endothelial cells suppress monocyte activation through secretion of extracellular vesicles containing anti inflammatory microRNAs. Blood. 2015;125:3202-12.

24. Huang H, Zhu J, Fan L, Lin Q, Fu D, Wei B, et al. MicroRNA profiling of exosomes derived from red blood cell units: implications in transfusion-related immunomodulation. Biomed Res Int. 2019;2019:2045915.

25. Doss JF, Corcoran DL, Jima DD, Telen MJ, Dave SS, Chi JT. A comprehensive joint analysis of the long and short RNA transcriptomes of human erythrocytes. BMC Genomics. 2015;16:952.

26. Jara EL, Muñoz-Durango N, Llanos C, Fardella C, González PA, Bueno SM, et al. Modulating the function of the immune system by thyroid hormones and thyrotropin. Immunol Lett. 2017;184:76-83.

27. Newton K, Dixit VM. Signaling in innate immunity and inflammation. Cold Spring Harb Perspect Biol. 2012;4(3):a006049.

28. Guo YJ, Pan WW, Liu SB, Shen ZF, XuY, Hu LL. ERK/MAPK signalling pathway and tumorigenesis. Exp Ther Med. 2020;19(3):1997-2007.

29. Burridge K. Focal adhesions: a personal perspective on a half century of progress. FEBS J. 2017;284(20):3355-61.

30. Downward J. Targeting RAS signalling pathways in cancer therapy. Nat Rev Cancer. 2003;3(1):11-22.

31. Sexton RE, Mpilla G, Kim S, Philip PA, Azmi AS. Ras and exosome signaling. Semin Cancer Biol. 2019;54:131-7.

32. Zeng Z, Wang K, Li Y, Xia N, Nie S, Lv B, et al. Down-regulation of microRNA-451a facilitates the activation and proliferation of CD4+ T cells by targeting Myc in patients with dilated cardiomyopathy. J Biol Chem. 2017;292(14):6004-13.

33. Qian M, Wang S, Guo X, Wang J, Zhang Z, Qiu W, et al. Hypoxic glioma-derived exosomes deliver microRNA-1246 to induce M2 macrophage polarization by targeting TERF2IP via the STAT3 and NF-KB pathways. Oncogene. 2020;39(2):428-42

34. Fang Y, Gao F, Hao J, Liu Z. microRNA-1246 mediates lipopolysaccharideinduced pulmonary endothelial cell apoptosis and acute lung injury by targeting angiotensin-converting enzyme 2. Am J Transl Res. 2017;9(3):1287-96.

35. Wan Y, Cui R, Gu J, Zhang X, Xiang X, Liu C, et al. Identification of four oxidative stress-responsive microRNAs, miR-34a-5p, miR-1915-3p, miR638, and miR-150-3p, in hepatocellular carcinoma. Oxid Med Cell Longev. 2017;2017:5189138.

36. Abdel-Latif GA, Elwahab AHA, Hasan RA, ElMongy NF, Ramzy MM, Louka $\mathrm{ML}$, et al. A novel protective role of sacubitril/valsartan in cyclophosphamide induced lung injury in rats: impact of miRNA-150-3p on NF-KB/MAPK signaling trajectories. Sci Rep. 2020;10(1):13045.

\section{Publisher's Note}

Springer Nature remains neutral with regard to jurisdictional claims in published maps and institutional affiliations. 